\title{
The USE OF WALKing MechanismS aS AN ADAPTIVE SuSPENSION OF A Planet Rover
}

\author{
Alexey Bogachev, Dmitriy Kuzmenko, Eugeny Lazarev \\ STC “ROCAD”, Lomanaya 11, Saint-Petersburg 196084, Russia
}

\begin{abstract}
The current paper presents a research on a possibility to use walking mechanisms of wheel-walking locomotion systems as an active adaptive suspension of a four-wheel planet rover. A two-arm walking mechanism with independent drive per each arm is investigated in the paper. The tasks of wheel-to-ground contact retention and keeping the vehicle body in horizontal position on the move are considered. An algorithm to control the arms of the walking mechanism as per the given tasks is proposed. The applied method of investigation and verification of the control algorithm workability is the object-oriented modeling. The modeling has been performed using sinusoidal surface. The obtained results of the locomotion modeling of a planet rover with passive and adaptive suspensions allow to confirm the possibility to use walking mechanisms as an active adaptive suspension of a planet rover.
\end{abstract}

Keywords: Planet rover; adaptive suspension; walking mechanism; object-oriented modeling
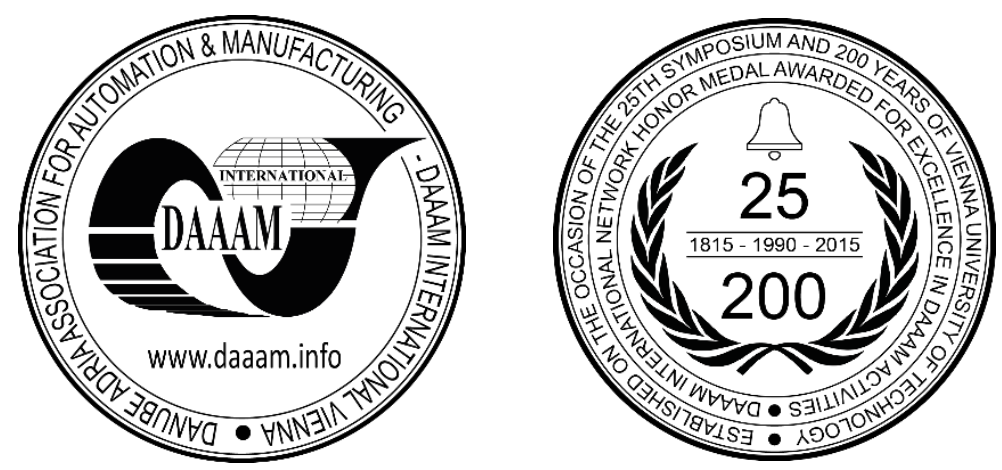

This Publication has to be referred as: Bogachev, A[leksey]; Kuzmenko, D[mitriy] \& Lazarev, E[ugeny] (2016). The Use of Walking Mechanisms as an Adaptive Suspension of a Planet Rover, Proceedings of the 26th DAAAM International Symposium, pp.0989-0996, B. Katalinic (Ed.), Published by DAAAM International, ISBN 978-3-902734-07-5, ISSN 1726-9679, Vienna, Austria

DOI: $10.2507 / 26$ th.daaam.proceedings. 139 


\section{Introduction}

Locomotion system of a planet rover is one of the most important parts of an on-planet activity mission. The wild surface of the planetary bodies is not generally favorable for the locomotion of the transport machines. For an example, the surface of Moon and Mars, which are very probable candidates for future planetary missions, has severe-tocross relief; many areas are covered with loose sands with low carrying capacity. Also, the gravity on the planets is different from the Earth gravity. Therefore, such specific operational conditions for the planet rovers impose certain requirements to the locomotion systems, which have to have high cross-country mobility, provide a possibility to adapt to various planet surface conditions, to ensure reliable operation of the rover and its on-board equipment during the mission.

\section{Description of planet rover locomotion system}

One of the possible ways to increase the mobility of the transport machines and planet rovers in particular, is the use of hybrid wheel-walking propulsors (primary movers). The idea to combine two classical propulsors (walking and rolling) was proposed in Russia in early 1970-ies and is very popular nowadays. The original idea to use wheel-walking locomotion system was to implement wheel-walking locomotion mode when negotiating loose soils with low carrying capacity. The theoretical background, various gaits and design examples are described in papers [2, 3]. In general, the present paper is based on the materials of paper [1], where a concept of an advanced planet rover locomotion system with four bearing-propulsive modules (BPM) is proposed. Each module includes two-arm (hip and shank) walking mechanism with independent drives of both arms and in-hub motor wheel. The paper examines a possibility to use such BPMs as an active adaptive suspension for this locomotion system. General view of the locomotion system and layout of the walking mechanism workspace are given in Fig. 1.

a

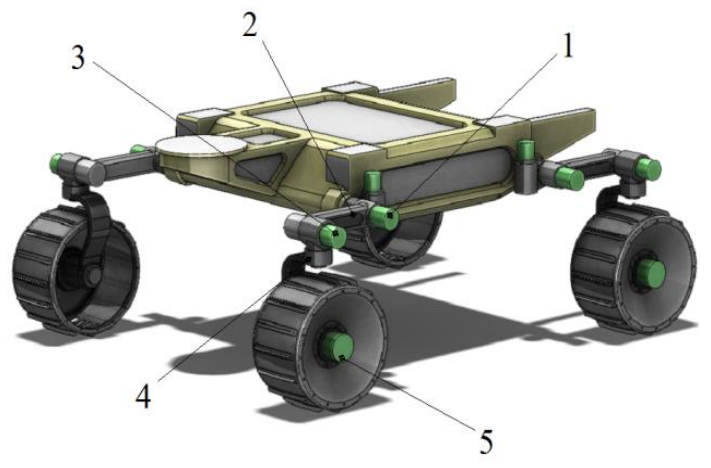

b

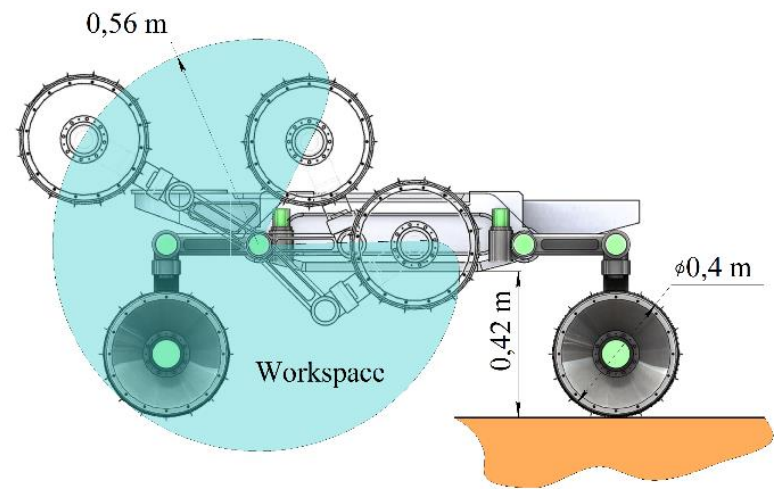

Fig. 1. Planet rover locomotion system:

a - general view of locomotion system: 1 - hip joint drive, 2 - hip, 3 - shank joint drive, 4 - shank, 5 - wheel drive; $\mathrm{b}$ - workspace of walking mechanism

The design of two-arm walking mechanism allows to considerably increase the working zone of wheel displacement relative to the rover body [1] and to implement any flat trajectory of the wheel axle within the working zone, including exact vertical displacement of the wheel. The ability to move the wheel axle in vertical plane is important for a transport machine, which moves over uneven terrain, where it might be necessary to either increase the ground clearance of the vehicle, or to move a separate wheel over an obstacle, while keeping the vehicle wheelbase unchanged irrespective of the wheel displacement. As an example, the paper [4] describes a robotic system SCARAB that was also developed for a planetary application and is able to realize wheel-walking locomotion mode. The authors of the paper [4] point out the ability of the SCARAB locomotion system to adapt to rough terrain relief. However, since SCARAB locomotion system has only single -arm walking mechanisms, there is no possibility to control the vertical position of the wheels without affecting the wheelbase.

\section{Formulation of the problem}

The purpose to use an adaptive suspension in off-road conditions is the preservation of permanent contact of all wheels with the ground, in order to ensure maximum tractive performance of the locomotion system. If one of the wheels of a four-wheel chassis looses the contact with the ground, and, at the same time, the center of mass of the chassis is not located within the stability triangle formed by other three wheel contact points, then the chassis will fall onto that wheel. The eventual impact of the chassis against the ground can cause malfunctioning of the rover on-board service and scientific equipment, or even mechanical damage of the chassis mechanisms.

The kinematic scheme proposed for the BPM in paper [1] allows to keep permanent contact of all wheels with the ground without alteration of the wheelbase and also to maintain optimal normal force in contact point of each wheel, 
which is achieved by equalizing the normal forces of the tractive wheels. These features enable the chassis to exert maximum traction force in hard off-road conditions.

Apart from the task to keep permanent contact of the wheels with the ground, the use of adaptive suspension allows to stabilize the horizontal position of the rover body on uneven terrain. The stabilization of the body position is especially important for ground piloted vehicles, because this provides better driving comfort for a human. A piloted planet rover is rather an exclusion (a unique example known so far is US lunar rover LRV), so the task to provide comfortable driving is not usually set for the designers of mobile planet robots. However, since the proposed locomotion system is to be used in robotic system "Astronaut Assistant", there might be the situations when the astronauts need to be transported by the robot with certain care. For instance, in an emergency situation when a disabled/wounded astronaut is transported to a medical aid, the excessive rocking of the moving robot due to rough terrain relief must be avoided.

Stabilization of the rover body position with respect to horizon allows to increase the capsizing stability of the planet rover in longitudinal and lateral directions. The elimination of the rover oscillation on the obstacles can provide better operational conditions for the rover's on-board service and scientific equipment. For an example, in order to measure the covered distance, the American Mars rovers, along with classical odometry based on wheel motor incremental encoders [5], are also equipped with a visual/optical odometry, based on stereo camera data [6]. The reason for this approach is that the wheel encoders measure only relative rotation of the wheels, the slippage of the wheels is not detected, which results in wrong estimation of actual displacement of the planet rover. The paper [6] presents the results of several tests, which show an error of $55 \%$ of wheel odometry when moving in sandy soil at a distance of 2,54 meter, while the error of visual odometry was under $1 \%$. In order to ensure proper functioning of visual odometry image analyzers, it requires to have $>60 \%$ overlap of two adjacent images, however, since the processing of two images takes about 2-3 minutes [6], it might happen that position of the stereo cameras could be changed because of an obstacle, so the required overlap is not maintained. The possibility to stabilize the position of the rover body with attached cameras while moving over the obstacles will allow to considerably reduce the probability of improper functioning of the visual odometry.

\section{Methodology and control algorithm}

In order to estimate the possibility to use walking mechanisms as an adaptive suspension, computer modeling method based on differential-algebraic equations has been applied.

The description of a system using this method is a universal tool that can be applied to investigate various dynamical physical processes with required accuracy [7]. In particular, an object-oriented modeling is used. Objectoriented modeling allows to preliminary estimate the workability of presumptive mechanical system control algorithm without developing physical models and experimental hardware.

The amount of vertical (normal) load on a wheel is directly affects the tractive force exerted by the wheel. A paper [8], that is using Bekker's theory of the wheel-to-ground interaction, shows the dependency of the tractive force vs. normal surface reaction. In order to control the normal reactions, the adaptive suspension control algorithm should be provided with data from force sensors in the wheels.

To stabilize the position of rover body, it is also necessary to introduce corresponding sensors - e.g. inclinometers, or gyroscopes [9].

A block diagram of proposed algorithm to control the coordinate $\mathrm{Z}$ (vertical axis) of an i-wheel axis is given in Fig.2.

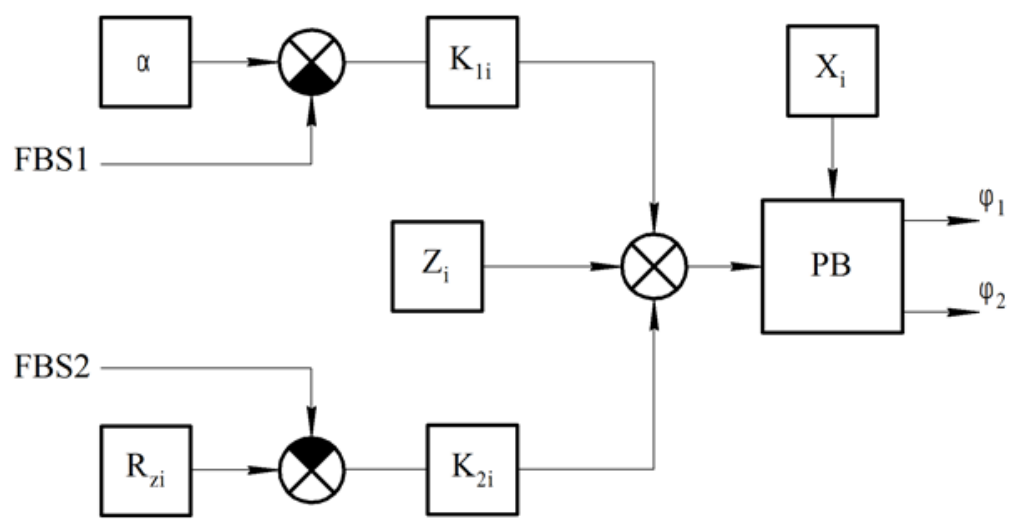

Fig. 2. A block diagram of proposed algorithm to control the coordinate $\mathrm{Z}$ of an $\mathrm{i}$-wheel axis $\alpha$-desired inclination angle of rover body in longitudinal direction, FBS1-feedback sensor of rover body inclination angle, $R_{z i}$-vertical load of an $i$-wheel, FBS2-feedback sensor of an $i$-wheel load, $K_{l i}, K_{2 i}$-correction coefficients of the errors of inclination angle and vertical load respectively, $Z_{i}$-nominal value of an $i$-wheel axis vertical coordinate, $X_{i^{-}}$ nominal value of an $i$-wheel axis horizontal coordinate, PB-processor block, $\varphi_{1}, \varphi_{2}$-joint rotation angles of walking mechanism 
Initially, the following parameters are to be set:

- $\quad \alpha$ - desired inclination angle of rover body in longitudinal direction;

- $\quad R_{z i}$ - vertical load of an $i$-wheel;

- $\quad Z_{i}$-nominal value of an $i$-wheel axis vertical coordinate.

Then, the values of these parameters are transferred to the adders (sector "subtraction" is color filled). The respective adders receive the data from feedback sensors FBS1 and FBS2. Coefficients $K_{l i}$, and $K_{2 i}$ are introduced in order to keep errors within working range of the wheel rotation axis. The value of the errors of inclination angle and vertical load are summed with nominal vertical axis coordinate and then proceed to the processor block. By solving reverse kinematics task for two-arm walking mechanism and knowing desired coordinates of the wheel rotation axis $(Z$ and $X)$, it is possible to define joint angles $\varphi_{1}$ and $\varphi_{2}$ that are required to realize these coordinates.

In order to verify the workability of proposed algorithm, a terrain surface with sinusoidal shape has been selected. The amplitude of the sinusoid is equal to wheel radius; sinusoid period is two-folds of the planet rover wheelbase. The assigned sinusoid parameter values are set so that front and rear wheels of the planet rover would had semi-period shift, which corresponds to the worst case, when rover body experiences maximum inclination without engaging adaptive suspension. The amplitude of the sinusoid was assigned to provide a reasonable ascent/descent angle that is tolerable for negotiation by the rover (for given sinusoid parameters, the angle is approximately $15^{\circ}$ ).

\section{Development of the model and simulation}

The use of object-oriented modeling methods allows to import from a CAD-software the 3D models of mechanical system components, including their mass-inertia properties, which improves the accuracy of the system analysis. Current analysis has been performed with assumption that all model elements are rigid bodies.

Since rotation axis of walking mechanism arms are located in common plane, then the model of the locomotion system can use flat contact model of two polygons to simulate interaction of the wheels with ground, i.e. the lateral forces were not taken into account, only normal and tangential wheel reactions were considered [10].

The terrain surface model was created such that sinusoids of the right and left boards of the rover were counterphase to each other and had small difference in the period. This decision allowed to observe anticipated impacts of the wheels that loose contact with the ground, however, in order to clearly see the impacts the modeling setup had to provide the rover body with all six degree of freedom. In opinion of the authors of the paper, this approach does not seriously affect the correctness of verification of the adaptation algorithm workability, because by the end of simulation of locomotion without adaptation the chassis drifted sideways by $1 / 4$ of the wheel width. in table 1 .

The modeling has been carried out assuming the locomotion system and test surface parameters, which are given

\begin{tabular}{|c|c|}
\hline \multirow{2}{*}{$\begin{array}{l}\text { Parameter } \\
\text { Total mass of the planet rover, } \mathrm{kg}\end{array}$} & Value \\
\hline & 1000 \\
\hline Length of the walking levers (hip/shank), m & $0,26 / 0,3$ \\
\hline Wheel diameter, $\mathrm{m}$ & 0,4 \\
\hline Ground clearance, $\mathrm{m}$ & 0,42 \\
\hline Wheel base, $\mathrm{m}$ & 1,27 \\
\hline Wheel track, m & 1,13 \\
\hline Amplitude of the sine-shape surface of the right and left chassis boards, $m$ & 0,2 \\
\hline Length of the half wave of the sine-shape surface of the right chassis board, $m$ & 1,45 \\
\hline Length of the half wave of the sine-shape surface of the left chassis board, $m$ & 1,8 \\
\hline \multirow{4}{*}{ Wheel-to-ground contact model } & 2 \\
\hline & 8000 \\
\hline & 0,8 \\
\hline & 9,81 \\
\hline
\end{tabular}

Table 1. The parameters of locomotion system and test surface 
In order to have better comparison, evaluation and result presentation, the model has been developed to provide a possibility to simulate both modes - with rigid suspension and with adaptive suspension.

Let us consider the locomotion of a planet rover with rigid suspension. Visualization window of the modeling process is given in Fig. 3.

After modeling the locomotion without adaptation of the walking mechanism arms (i.e. the joints of arms were rigidly fixed) the values of the normal wheel reactions were obtained. Fig. 4 shows values of the normal wheel reactions.

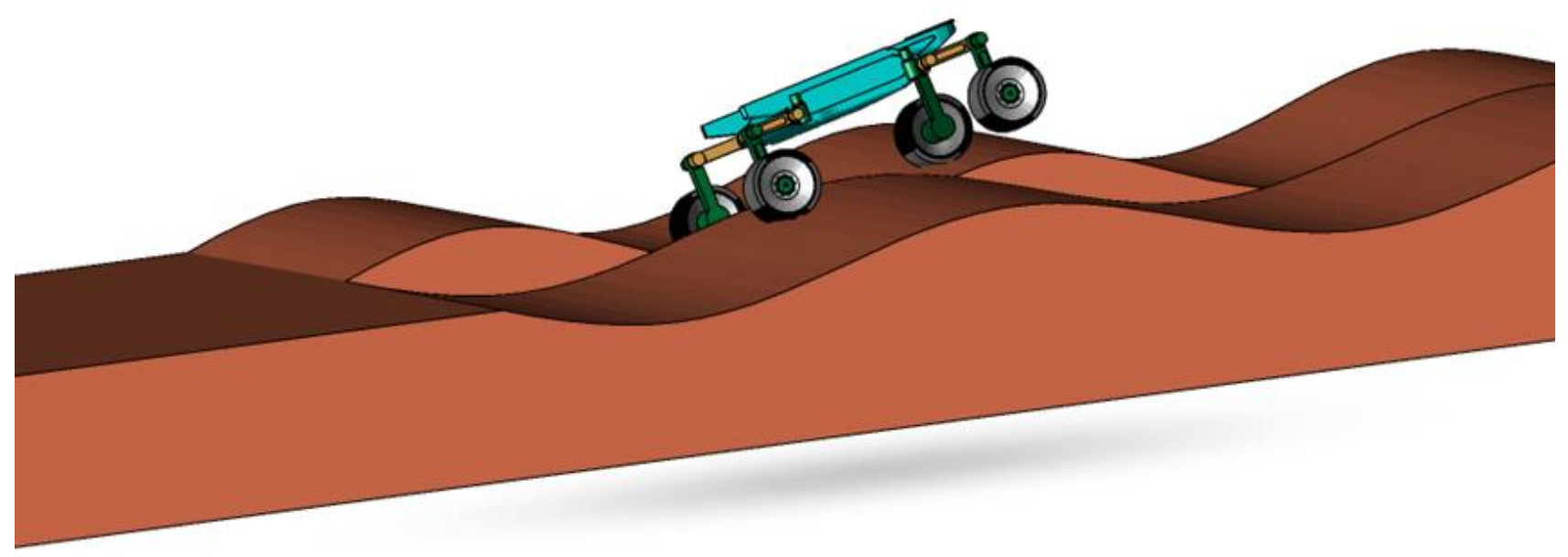

Fig.3. Visualization window of the modeling process of locomotion with rigid suspension
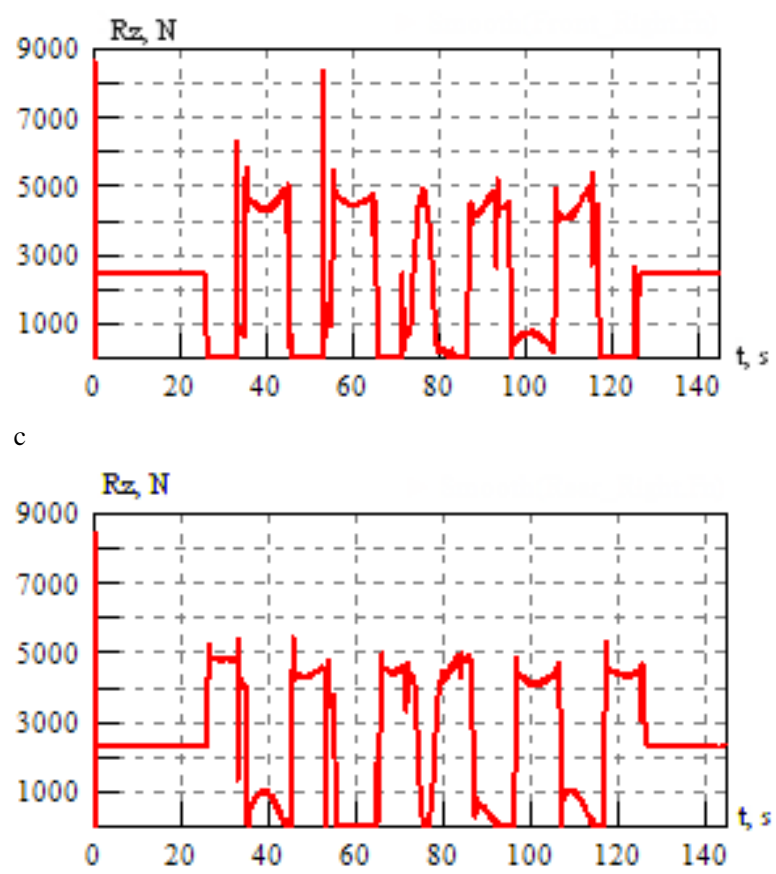

b

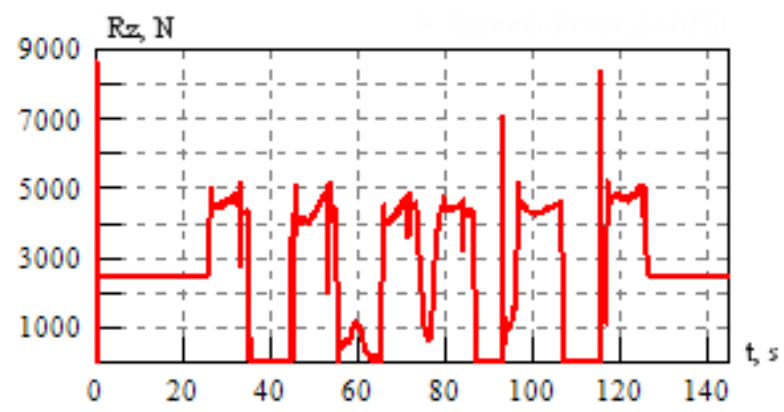

d

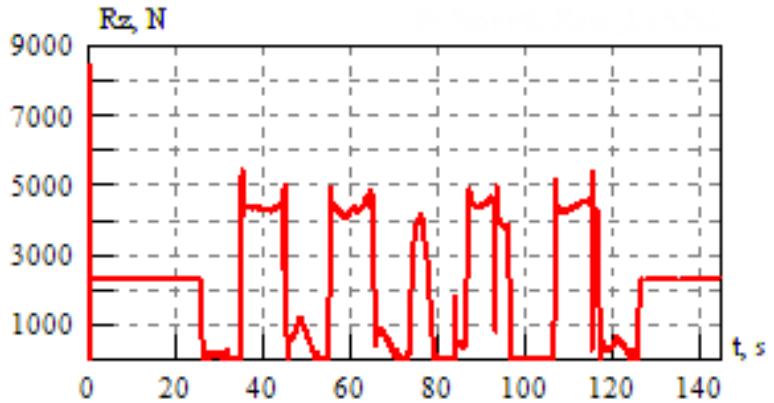

Fig. 4. The plots of the dependencies of the normal wheel reactions vs. modeling time for the chassis with rigid suspension:

a) front right wheel; b) front left wheel; c) rear right wheel; d) rear left wheel

The graphs in Fig. 4 show that for a locomotion system with rigidly fixed walking arms there are both anticipated impacts (peaks in the graphs) of the wheels against the ground and the events when a wheel is in air (zero normal reactions). Horizontal parts of the graphs show normal reactions of the wheels when rover moves on even flat surface.

Let us consider a case when the rover moves over the same terrain surface, but with use of the adaptation algorithm as described above. The modeling results are given in Fig.5-6. 


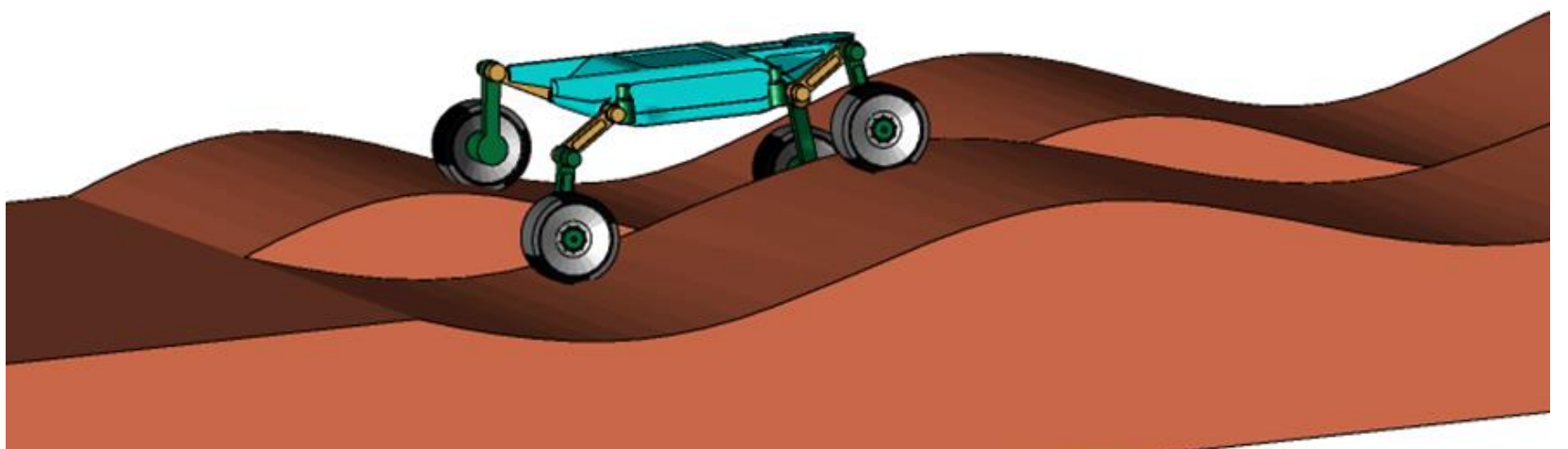

Fig. 5. Visualization window of the modeling process of locomotion with adaptive suspension
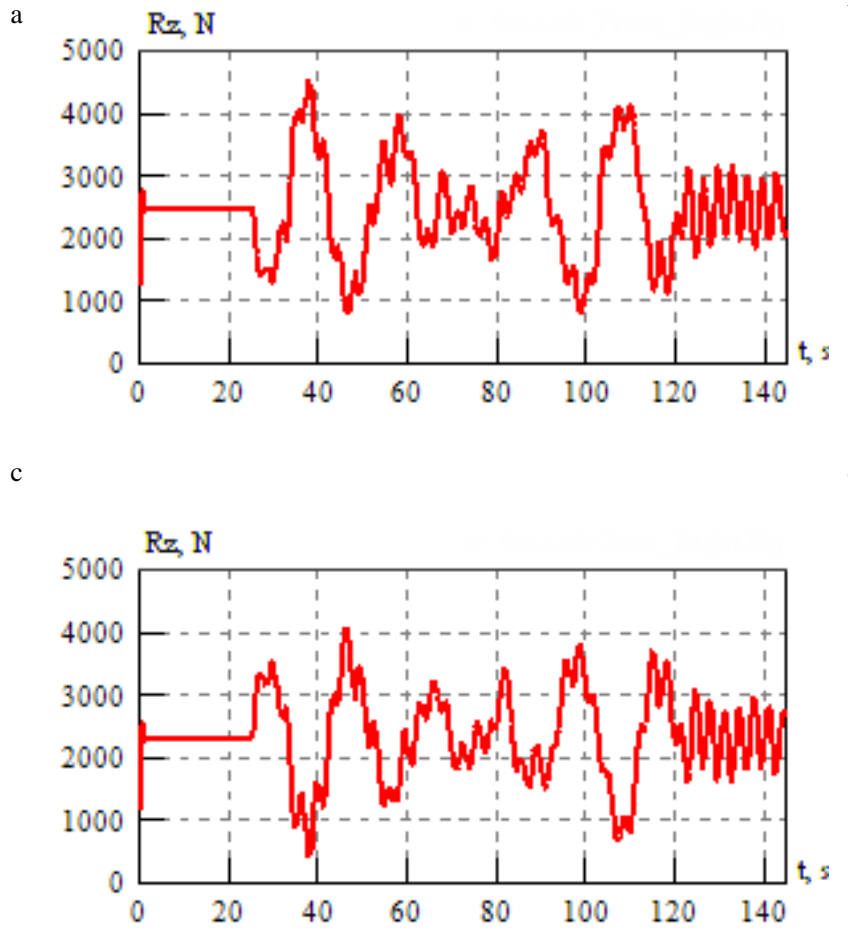

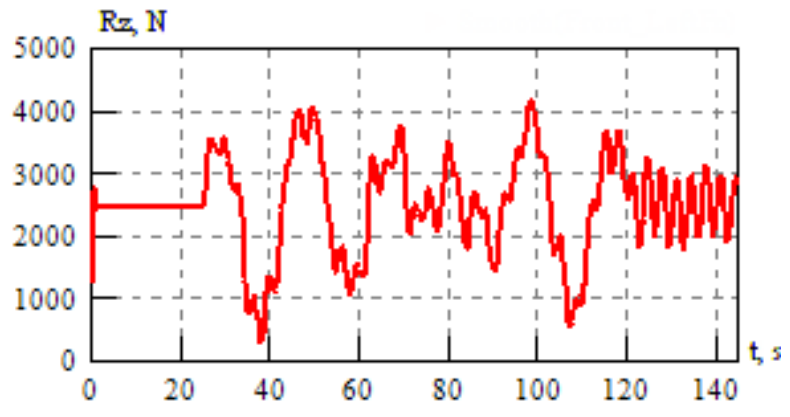

d

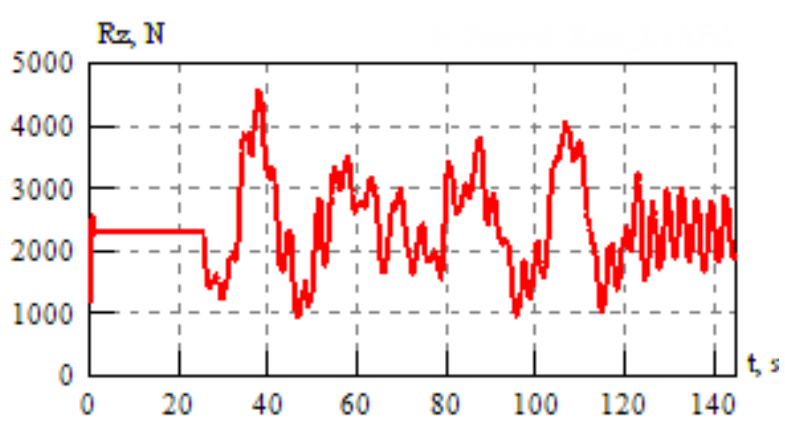

Fig. 6. The plots of the dependencies of the normal wheel reactions vs. modeling time for the chassis with adaptive suspension:

a) front right wheel; b) front left wheel; c) rear right wheel; d) rear left wheel

The modeling results given in the Fig. 6 show that the wheels are in permanent contact with the ground, there are no impacts of the wheels against the ground. Also, the angular oscillation of the rover body in longitudinal plane is considerably reduced compared with the oscillation of the rover with fixed walking mechanism arms (Fig. 7). 


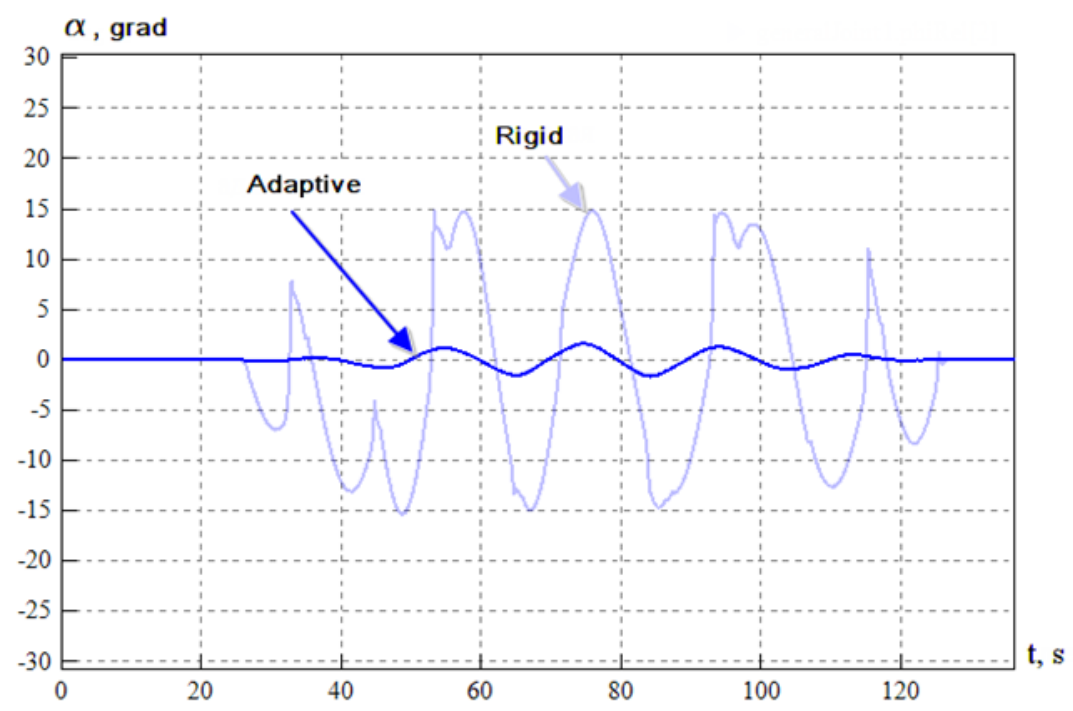

Fig. 7. Dependence of rover body inclination angle in longitudinal plane vs. modeling time

\section{Conclusion}

The use of the wheel-walking locomotion systems considerable improves the mobility of the transport machines, and, in particular, the planet rovers on loose soils with low cohesion. However, this solution negatively affects the locomotion over complex relief with various vertical obstacles: loss of the wheel contact with the ground, impacts of the wheels against the ground, excessive oscillation of the rover body. To eliminate these drawbacks, it was proposed to use walking mechanisms as an adaptive suspension. A corresponding control algorithm for the adaptive suspension has been developed. The algorithm is using the data from sensors of wheel vertical loads and rover body inclination angles. The verification of the algorithm workability has been performed with the help of object-oriented modeling method. The obtained results allow to conclude that the use of walking mechanisms of wheel-walking locomotion systems as an active adaptive suspension of a planet rover is possible. Proposed walking arm control algorithm allows to keep permanent contact of the wheels with uneven terrain, which increases the mobility of the planet rover in hard off-road conditions, and provides a possibility to maintain horizontal position of the rover body, which can be used to improve the performance of on-board scientific and navigational equipment on the move.

As the next steps in the research on further development of the control algorithm of walking mechanism it is panned to implement PID controller, to develop a more precise model of the interaction of the wheel with ground, to add to the joint models the physical properties of electric motors and gears, to conduct experimental investigation of the control algorithm.

\section{Acknowledgements}

The applied scientific research is carried out with the support of the Russian Ministry of Education and Science within the project 14.576.21.0050 "Creation of scientific and technical solutions in the field of development of robotic tools for space applications to provide planetary missions", having a unique identifier of applied research RFMEFI57614X0050.

\section{References}

[1] Malenkov M.I., Volov V.A., Guseva N.K., Lazarev E.A. "Analysis on mobility of the Mars rovers to support the developments of locomotion systems and control algorithms of the planet rovers of new generation " - News of South Federal University. Technical Science. Publisher: South Federal University.

[2] Kahjukalo I.F. A walking principle in propulsors of the transport machines. Wheel-walking propulsor // Planet rovers / Under edition of Kemurdjian A.L. - Moscow, Mashinostroenie, 1082. - p. 65-107.

[3] Kahjukalo I.F. Wheel-walking propulsor as a mean to enhance the mobility // Locomotion over the terrains of Moon and planets / Under edition of Kemurdjian A.L. - Moscow, Mashinostroenie, 1086. - p. 151-185.

[4] Bartlett P., Wettergreen D., Whittaker W.L. The SCARAB rover as designed for lunar science and resource exploration // Lunar and Planetary Science Conference XXXIX. - 2008.

[5] Odometry // a collective blog Robokraft: [site]. [2012]. URL: http://robocraft.ru/blog/technology/736.html (date of addressing: 28.07.2013).

[6] Visual Odometry on the Mars Exploration Rovers. BY YANG CHENG, MARK W. MAIMONE, AND LARRY MATTHIES 
[7] Modeling and virtual prototyping: a tutorial / I.I. Kosenko [and others]. - Moscow: Infa-M Alpha-M Unikum Service, 2015, - 176 p. Technological service. - Magistratura. - ISBN 978-5-98281-280-3. — ISBN 978-5-16005167-3.

[8] Xiao-liu Yu ,Lei Fang ,Jin-fu Liu. Interaction Mechanical Analysis between the Lunar Rover Wheel-Leg Foot and Lunar Soil. 2012 International Workshop on Information and Electronics Engineering (IWIEE), Procedia Engineering 29 (2012) 58-63.

[9] Radek Votrubec. Stabilization of Platform using Gyroscope. 24th DAAAM International Symposium on Intelligent Manufacturing and Automation, 2013, Procedia Engineering 69 ( 2014 ) 410 - 414.

[10] Greiner, G., Hormann, K.: Efficient Clipping of Arbitrary Polygons, Computer Graphics Group, University of Erlangen, July 2011. 